\title{
On Approach of Organic Agriculture Growth Mode Change in China
}

\author{
Xin-min ZHANG \\ Heilongjiang Bayi Agricultural University, Daqing, China \\ qchzxm@126.com
}

Keywords: Organic agriculture, Growth mode, Transformation path.

\begin{abstract}
In recent years, the field of organic agriculture is widely emphasized with continuous expansion of organic agriculture production scale in China. However, there is still larger development space from the perspective of practical layer, there are still many problems in the aspects of production, consumption, policy, etc., which should be solved. In the study process of the paper, the author will discuss on the basis of the field, and related factors of organic agriculture production will be combined for in-depth discussion, and development countermeasures are put forward.
\end{abstract}

\section{Introduction}

Development of organic agriculture in China shows a state of lag from a global perspective. It also brings impact on farmer income increase, which is unfavorable to solve the issues of agriculture, farmer and rural area. On the basis, the field is studied, and it has very important significance in the aspects of theory and practice.

\section{Main mode of organic agriculture development in China}

\section{Government-oriented development}

Organic agriculture is an important public cause to realize sustainable development of agriculture, wherein agriculture, environmental protection, land and resources as well as other departments should cooperate and contact mutually. On the basis, it can be concluded that government orientation becomes the most common mode in the process of organic agriculture development. Government can provide support in many aspects for organic agriculture, which is related to policy, funding, technology and other aspects. More farmers and enterprises are mobilized to participate in the process through propaganda, demonstrations and other forms. It has important function to change agricultural development mode. For example, China Ningxia Hui Autonomous Region expanded the scale of organic agricultural production enterprises from the aspect of policy since 2010. Annual yield of organic rice in the demonstration base has been up to 3260t. Total output of rice, crabs, duck and fish is 24.26 million Yuan. Compared with conventional rice, income per ton is increased by more than 5250 Yuan. It should be admitted that economic benefits are improved to certain degree, and social benefits are also highlighted.

\section{Development driven by leading enterprises}

In organic agriculture production process, some leading enterprises focus on production, processing and sales of one or several organic products in order to expand the influence of the enterprise and increase the production efficiency to certain level, thereby realizing organic combination with production base and farmers to certain extent. It is so-called integrated management pattern. All economic communities can share the risks and enjoy benefits under such management mode. Enterprises can suitably provide the base and farmers with certain support in organic agriculture, and they can provide services comprehensively. Farmers should deliver and sell high-quality products to enterprises at fixed time and with fixed quantity according to regulation in the contract, then the company should process the products and sell the finished products. For example, there are special tea companies in Guangxi Zhuang Autonomous Region. They are responsible for producing organic tea with more prominent brand benefits. In the region, farmers 
participating in organic tea production have average annual income of more than 8900 Yuan. It can be concluded that such farmers have per capita annual income three times higher than that of farmers in the whole county.

\section{Characteristic industrial upgrading development}

Organic agriculture has certain requirement on improving the quality of characteristic agricultural products. Optimization and upgrading of product structure are emphasized, thereby resisting risks. Therefore, it has very important role in the aspect of expanding export of agricultural products. Since it reflects certain economic benefits and ecological benefits since the generation, characteristic industry upgrading drives organic agriculture, and it has become a new approach of their development. Shandong Province in China obtained more prominent achievements in the aspect. Jinxiang County of Shandong Province implements industrialized development on garlic cultivation. Industry transformation is realized especially under the background that domestic agriculture production is increasingly fierce in China, organic production elements are integrated, which has very important significance for regional agriculture development. Till present, large-scale trend of garlic planting has been realized in the county, the demonstration base area is $1333.33 \mathrm{hm} 2$, including high standard demonstration base of $133.33 \mathrm{hm} 2$. It shows stronger stability and competitiveness in the aspects of export and price.

\section{Development driven by environmental protection}

Many cities in China have improved ecological farming in order to fundamentally solve problem of ecological environment protection, and promote development of organic agriculture Pig farm in Dawa County of Liaoning is typical, virtuous circle of 'fertilizer-pond-paddy field-water purification' is realized as shown in figure 1.

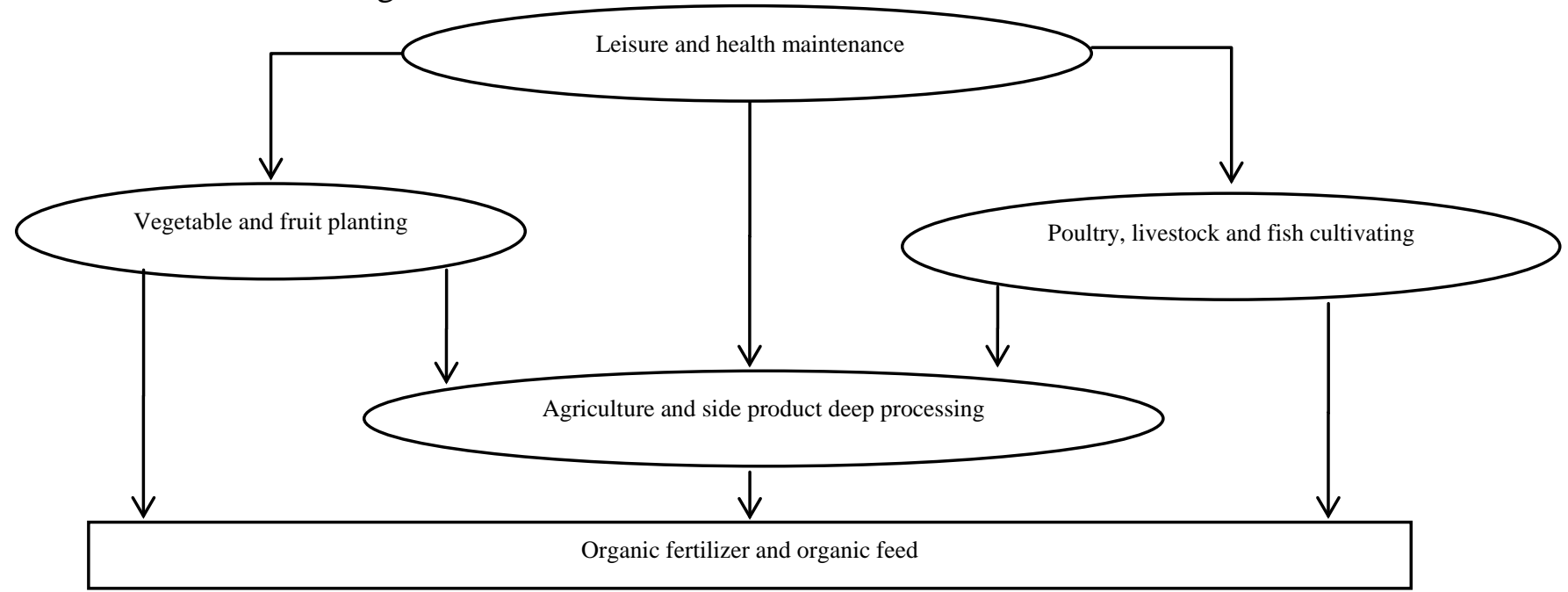

Fig. 1 Cyclic process of organic agriculture

In the process, energy and materials can exert maximum value on one hand, the harm of dirty water and flies due to pig farming can be solved to certain extent on the other hand.

\section{Present situation and existing problems of organic agriculture growth mode in China}

\section{Analysis on present situation}

Organic agriculture in China was started late compared with western countries. However, the development speed is faster, organic agriculture is driven for appearance under export demand at the beginning, and it mainly aims at Japan, European Union and the United States. Therefore, 95\% organic agriculture products in China are exported to international market at the initial stage. However, organic agriculture market is wider and wider in China in recent years, it has certain influence in China, and concrete condition is shown in figure 2.

Organic agriculture production bases in China are mostly distributed in eastern coastal region and the northeast provinces from the perspective of regional distribution. Three provinces in northeast and Inner Mongolia are the largest from two aspects of quantity and area. However, the southeast 
coastal area has certain advantage in the aspect of processing technology. So far, there are more categories of organic agricultural products in China. In addition, most products belong to primary raw materials, and processed products are relatively few.

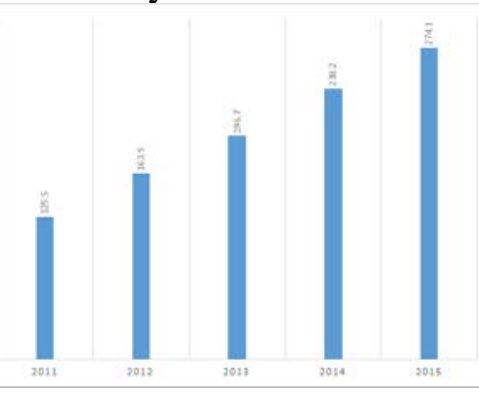

Fig. 2 The size of the market about organic agricultural products industry from2011-2015

Statistics of relevant departments in China show that GDP of organic agricultural products in China is 200 million Yuan or so in 2004 in China and total value of exported organic products is 100 million Yuan. China growth mode of organic agriculture is changed dramatically with continuous improvement of people's production and living standard. Domestic consumption is much more than export. In the future, China organic agriculture will enter a relatively rapid growth stage become China organic agriculture market has formed certain scale on one hand, people's environment and food safety awareness are growing constantly on the other hand. Government attaches more and more importance to organic agriculture. The development of organic agriculture is not only beneficial for protecting rural ecological environment, but also can utilize existing resources efficiently, thereby ensuring food quality and safety, and protecting people's health in body and mind. It can be concluded that development of organic agriculture has become the focus with attention in the society. Health and environmental consciousness are unceasingly enhanced aiming at consumers, and greater importance is attached to production and development of organic agriculture. In many areas, related development plans of regional organic agriculture are also prepared, supporting policies are published, thereby providing convenient conditions for development organic agriculture.

\section{Existing problems}

Fund shortage of agriculture scientific research investment and agriculture technology popularization. Support of technology and funds is necessary in the process of organic agriculture growth mode transformation. Development experience of developed countries shows that the United States is the first country investing in the scientific research cost as early as 1980s. The investment was increased by one time every ten years subsequently. However, actual situation in China shows that it started to pay more and more attention since the new century. Currently, total output of agriculture accounts for higher proportion in China organic agricultural research expenditure. However, research, development and promotion funds are not sufficient as shown in figure 3.

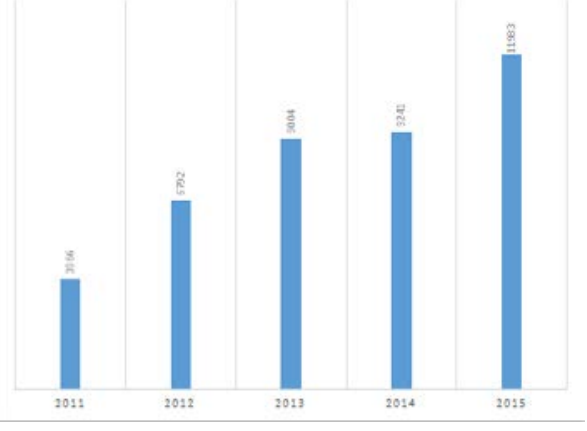

Fig.3 Fixed assets investment of organic agriculture from 2011-2015

Smaller production scale and insufficient high-quality brand. Currently land system in China is still mainly based on household contract responsibility system. Each farmer occupies smaller arable land area from the perspective of farmers. It is difficult to former certain scale. Production of farmers will be affected by surrounding environment. If farmer individuals are merely relied for promotion, it is obvious that certain expectation effects can not be realized. In addition, per capita arable land is less in China. Meanwhile, organic agriculture is developed in mountainous area under 
most circumstances. However, it is difficult to realize large-scale production in local land. It is not convenient to implement large-scale production. Therefore, certain difficulty can be brought to organic agriculture mechanization and standardization implementation. Production cost of organic agriculture is constantly increased, thereby indirectly affecting market price of organic agriculture.

Insufficient accumulation of organic agriculture human capital. The cultural level and technical quality of farmers have direct influence to the development of organic agriculture. China farmer's quality is improved actually compared with that in the early days after foundation of new China. However, it is not substantially changed. At present, farmers are trained in the aspect of practical technology in China. However, improvement of farmers' culture level is ignored, and long-term benefits of farmers are not emphasized.

Insufficient high-quality brand. It is obvious in actual investigation that though high-quality products have been developed among Chinese farmers and a part of agricultural cooperatives at present, brand consciousness is insufficient, therefore market operation ability should be improved, and product quality is questioned. In recent years, China network information publicity effort is constantly increased; farmers' market values have been changed dramatically. However, phenomenon of mud and sand flowing is common. Many producers focus on personal interests merely and ignore overall social efficiency, and it is difficult to achieve brand value.

\section{Approach of transforming organic agriculture growth mode in China}

Increasing income of organic agriculture science and technology. We should pay high attention to scientific investment in order to maximally ensure steady growth of agricultural production and avoid the influence of bad weather. It is also important basis to realize organic agricultural production intensive growth in China. In the future, national expenditure in agricultural technology research and technology promotion should be increased on one hand; increase of agriculture realistic productivity also should be focused on the other hand with more and more prominent trend of agricultural science and technology progress. Secondly, application in the aspect of China organic agriculture seed improvement should be maintained continuously, and advantages in the aspects of fertilizer, pesticides and other aspects should be utilized as far as possible. Traditional agricultural technology in China can be fully emphasized and carried forward. It can be combined with modern science and technology together. Modern biological technology, atomic energy technology, remote sensing technology and other advanced technology still should be utilized, thereby ensuring transformation of organic agricultural growth mode in the aspect of technology.

\section{Gradually realizing large-scale management of organic agriculture}

Mechanization production mode of organic agriculture is realized, thereby directly affecting large-scale management of agriculture. It is difficult to form mechanization of agriculture under the mode of family management, especially in China with less population, more land and lower urbanization level, and a process is required for agriculture large-scale management and mechanization. Therefore, it is necessary to create good condition for large-scale management of organic culture under current background. Concretely speaking, land market should be established gradually, which should be perfected effectively, thereby ensuring flow of land use right. The government should regulate from macro point of view. Transfer of rural surplus labor force should be further strengthened. Therefore, in-depth development of agriculture resources can be realized by relying on small towns.

\section{Improving accumulation of organic agriculture human capital}

Progress and development of agricultural science and technology must depend on human capital support. It is also an important factor to realize agriculture growth mode transformation. Currently, construction and accumulation of human capital in organic agriculture should be strengthened as far as possible. Agriculture practical training and improvement should be emphasized. Rural labor can be cultivated into various specialized talents. In addition, farmers' cultural quality should be strengthened constantly, thereby greatly improving agricultural labor force culture level. 


\section{Strengthening construction of organic agriculture brand}

Corresponding system should be formulated aiming at organic agricultural product brand construction. Therefore, sustainable development of organic products can be actually guaranteed. In the process of brand construction, information smoothness should be focused, organic product production enterprises should provide actual and effective information as far as possible, thereby firmly mastering market dynamic state and industry development level, and the situation of asymmetric information in the market can be fundamentally solved.

\section{Conclusion}

In the research process of the paper, the author regards organic agriculture growth condition as research background. Problems in organic agriculture growth mode transformation process are analyzed, thereby putting forward development approach, including policy, technology, human capital, etc. Research in the paper is in line with requirements of Chinese situation at present with certain operational value and significance.

\section{Acknowledgement}

This research was financially supported by the planning topic of philosophy and social sciences of Henan Province in 2013 (2013BJJ04) and the soft science programs of Henan Province in 2016 (162400410012).

\section{References}

[1] Cai Jian, Li Xiaoqi. Approach selection of agricultural growth mode transformation-with Guangdong Province as an example. Journal of Jiangxi Agricultural University (Social Science Edition), 2013,03:281-288.

[2] Nie Yazhen. Analysis on approach of agricultural growth mode transformation in backward area. Journal of Theory, 2015,02:176-177.

[3] Li Shengming. On approach dependence of economic growth mode transformation in China national autonomous areas. Economic And Social System Comparison, 2012,04:178-180.

[4] Sun Ling. Approach selection of agricultural economic growth mode transformation in China western region. Theory and Reform, 2014,03:101-103.

[5] Zhang Zuyan. Research on China rural economic growth mode transformation and direction. Journal of Xianning College, 2014,09:8-9+20.

[6] Bai Xuerui. Analysis on factors restricting agricultural growth mode transformation in China. Journal of Economic Study, 2015, 10: 32-34.

[7] Zhu Yutao, Zhao Junyan, Zhao Huifeng. Approach selection and countermeasures of animal husbandry growth mode transformation in China. Market Modernization, 2014,03:280.

[8] Liu Zhanqing. Research on agricultural economic growth mode transformation research in China. China Rural Observation, 2012,03:45-51.

[9] Zhu Yutao, Zhao Junyan, Zhao Huifeng. Research on approach selection and countermeasure of animal husbandry growth mode transformation in China. Heilongjiang Animal Husbandry and Veterinary Market Modernization 2015,08:16-17.

[10] Jiang Yongmu. Necessary acceleration of transformation of China agricultural growth mode. Journal of Sichuan University (Philosophy and Social Sciences Edition) 2013,02:3-7. 